\title{
Statistical Morphological Disambiguation for Agglutinative Languages
}

\author{
Dilek Z. Hakkani-Tür, Kemal Oflazer, Gökhan Tür \\ Department, of Computer Engineering, \\ Bilkent University, \\ Ankara, 06533, TURKEY \\ \{hakkani,ko, tur\}@cs.bilkent.edu.tr
}

\begin{abstract}
In this paper, we present statistical models for morphological disambiguation in Turkish. Turkish presents an interesting problem for statistical models since the potential tag set size is very large becautse of the productive derivational morphology. We propose to handle this by broking up the morhosyntactic tags into inflectional groups, ach of which contains the inflectional features for each (intermediate) derived form. Our statistical models score the probability of cach morhosyntactic tag by considering statistics over the individual inflection grouss in a trigram model. Among the three models that we have developed and testod, the simplest model ignoring the local morphotactics within words performs the best. Our best trigram model performs with $93.95 \%$ accuracy on our test data getting all the morhosyntactic and semantic foatures correct. If we aro just interested in syntactically rolevant, fatures and ignore a very small set of semantic features, then the accuracy increases to $95.07 \%$.
\end{abstract}

\section{Introduction}

Recent advances in computer hardware and availability of very large corpora have made the application of statistical technicues to matural language processing a feasible and a very appealing researeh arca. Many useful results have been obtained by applying these techniques to English (and similar languages) - in parsing, word scnse disambignation, part-of-specch (POS) tagging, spech recognition, etc. However, languages like Tukish, Czech, Hungarian and Finnish, display a substantially different behavior than English. Unlike English, these languages have agglutinative or inflective morphology and relatively free constituent order. Such languages have received litile previous attention in statistical processing.

In this paper, we present our work on modeling Turkish using statistical methods, and present rcsults on morphological disambiguation. The methods developed here are certainly applicable to other agghtinative languages, especially those involving productive derivational phenomena. The paper is organized as follows: After a brief overview of related previous work, we summarize relevant aspects of Turkish and present details of various statistical nockels for morphological disambiguation for 'Turkish. Wo then present results and analyses from on experiments.

\section{Related Work}

There has been a large number of studies in lagging and morphological disambiguation using various techniques. POS tagging systems have used cither a statistical or a rule-based approach. In the statistical approach, a large corpus has been used to train a probabilistic model which thon has boen used to talg new text, assigning the most likely tag for a given word in a given context (e.g., Church (1988), Cutting et al. (1992)). In the rule-based approach, a large number of hand-crafted linguistic constraints are nsed to oliminate impossible tags or morphological parses for a given word in a given context (Karlsson et al., 1.995). Buill (1995a) has presented a transformation-based leaning approach, which induces disambiguation rules from tagged corpora.

Morphological disambiguation in inflecting or agglutinative languages with complex morphology involves more than determining the major or minor parts-of-specech of the lexical items. Typically, morphology marks a number of inflectional or derivational features and this involves ambiguity. For instance, a given word may be chopped up in different ways into morphemes, a given morpheme may mark different features depending on the morphotactics, or lexicalized variants of derived words may interact, with productively derived versions (see Oflazer and Tïr (1997) for the different kinds of morphological ambiguities in Turkish.) We assume that all syntactically relevant features of word forms have to be determined correctly for morphological disambiguation.

In this context, there have been some interesting previous studies for different languages. Lcvinges: et al. (1995) have reported on an approach that learus morpholoxical probabilities from an untagged corpus and have used the resulting information in 
morphological disambiguation in Hebrew. Hajic and Hladká (1998) have used maximum entropy modcling approach for morphological disambiguation in Crech. Ezciza et al. (1998) have combined stochastic and rule-based disambiguation methods for Basque. Megyesi (1999) has adapted Brill's POS tagger with extended lexical tomplates to Hungarian.

Previous approaches to morphological disambiguation of Turkish text had employed a constraintbased approach (Oflazer and Kuruöz, 1994; Oflazer and Tür, 1996; Oflazer and Tür, 1997). Although results obtained earlier in these approaches were reasonable, the fact that the constraint rules were hand crafted posed a rathor serious impediment to the gencrality and improvement of these systems.

\section{Turkish}

Turkish is a free constituent order language. The order of the constituents may change freely according to the discourse context and the syntactic role of the constituents is indicated by their case marking. Turkish has agglutinative morphology with productive inflectional and derivational suffixations. The number of word forms one can derive from a Turkish root form may be in the millions (Hankanner, 1989). Hence, the number: of distinct word forms, i.e., the vocabulary size, can be very large. For instance, Table 1 shows the size of the vocabulary for 1 and 10 million word corpora of Turkish, collected from online newspapers. This large vocabulary is the reason

\begin{tabular}{|l|c|}
\hline Corpus size & Vocabulary size \\
\hline $1 \mathrm{M}$ words & 106,547 \\
$10 \mathrm{M}$ words & 417,775 \\
\hline
\end{tabular}

Table 1: Vocabulary sizes for two Turkish corpora.

for a serious data sparseness problem and also significantly increases the number of parameters to be estimated even for a bigram language model. The size of the vocabulary also causes the perplexity to be large (although this is not an issue in morphological disambiguation). Table 2 lists the training and test set perplexities of trigram language models trained on 1 and 10 million word corpora for Turkish. For cach corpus, the first column is the perplexity for the data the language model is trained on, and the second column is the perplexity for previously unsecn test data of 1 million words. Another major reason for the high perplexity of Turkish is the high percentage of out-of-vocabulary words (words in the test data which did not occur in the training data); this results from the productivity of the word formation process.

\begin{tabular}{|l|c|c|}
\hline $\begin{array}{l}\text { Training } \\
\text { Data }\end{array}$ & $\begin{array}{c}\text { Training Set } \\
\text { Perplexity }\end{array}$ & $\begin{array}{c}\text { Test Set (1M words) } \\
\text { Perplexity }\end{array}$ \\
\hline $1 \mathrm{M}$ words & 66.13 & 1449.81 \\
$10 \mathrm{M}$ words & 94.08 & 1084.13 \\
\hline
\end{tabular}

Table 2: The perplexity of Turkish corpora using word-based trigram langnage models.

The issue of large vocabulary brought, in by productive inflectional and derivational processes also makes tagset design an important issue. In languages like English, the number of POS tags that can be assigned to the words in a text is rather limited (less than 100, though some researchers have used large tag sets to refine gramularity, but they are still small compared to Turkish.) But, such a finite tagset approach for languages like Turkish may lead to an inevitable loss of information. The reason for this is that the morphological features of intermediate derivations can contain markers for syntactic relationships. Thus, leaving out this information within a fixed-tagset scheme may prevent crucial syutactic information from being represcnted (Oflazer of al., 1999). For example, it is not clear what POS tag should be assignod to tho word sağlamlastamak (below), without losing any information, the catcgory of the root (Adjective), the final category of the word as a whole (Noun) or one of the intermediate categories (Vorb). ${ }^{1}$

sağlam + lass + tır + mak

sağlam+Adj ${ }^{\wedge} D B+\underline{V e r b}+B e c o m e-D B$

+ Verb $+\overline{\text { Caus }}+$ Pos ${ }^{\wedge} \mathrm{DB}+$ Noun + Inf $+\mathrm{A} 3 \mathrm{sg}+\mathrm{Pnon}+\mathrm{Nom}$

to cause (something) to become strong/

to strengthen/fortify (something)

Ignoring the fact that the root word is an adjective may sever any relationships with an adverbial modifier modifying the root. Thus instead of a simple POS tag, we use the full morphological analyses of the words, represented as a combination of features (including any derivational markers) as their morphosyntactic tags. For instance in the example above, we would use everything including the root form as the morphosyntactic tag.

In order to alleviate the data sparseness problem we break down the full tags. We represent each word as a sequence of inflectional groups (IGs hercafter), separated by "DBs denoting derivation boundaries, as described by Oflazer (1999). Thus a morphological parse would be represented in the following gencral form:

\footnotetext{
${ }^{1}$ The morphological features other than the POSs are: +Become: become verb, +Caus: causative verb, +Pos: Positive polarity, +Inf: marker that derives an infinitive form from a verb, +\$3sg: $3 \mathrm{sg}$ number-person agreement, +Pnon: No possessive agreement, and +Nom: Nominative case. -DB's mark derivational boundaries.
} 


\begin{tabular}{|l|r|r|}
\hline & Possible & Observed \\
\hline Full Tags (No roots) & $\infty$ & 10,531 \\
\hline Inflectional Groups & 9,129 & 2,194 \\
\hline
\end{tabular}

Table 3: Numbers of Tags and IGs

$\operatorname{root}+\mathrm{IG}_{1}{ }^{\wedge} \mathrm{DB}+\mathrm{IG}_{2}{ }^{\wedge} \mathrm{DB}+\cdots \sim{ }^{\sim} \mathrm{DB}+\mathrm{IG}_{n}$

where $\mathrm{IG}_{i}$ denotes relevant inflectional features of the inflectional groups, including the part-of-spech for the root or any of the derived forms.

For exanple, the infinitive form sağlamlastmmal given above would be represented with the aldjective reading of the root sağlam and the following 4 IGs:

1. Adj

2. Verb+Become

3. Verb+Caus+Pos

4. Noun+Inf + A3sg+Pnon+Nom

Table 3 provides a comparison of the number distinct full morphosyntactic tags (ignoring the root words in this case) and IGs, generatively possible and obscrved in a corpus of $1 \mathrm{M}$ words (considering all ambiguities). One can sec that the number obsorved full tags ignoring tho root words is very high, significantly highor than quoted for Crech by Hajic and Hladká (1998).

\section{Statistical Morphological Disambiguation}

Morphological disambiguation is the problem of finding the corresponding sequence of morphological parses (including the root), $T=t_{1}^{n}=t_{1}, t_{2}, \ldots, t_{n}$, given a sequence of words $W=w_{1}^{n}=w_{1}, w_{2}, \ldots, w_{n}$. Our approach is to model the distribution of morphological parses given the words, using a hidden Markov model, and then to seok the variable $T$, that. maximizes $P(T \mid W)$ :

$$
\begin{aligned}
\underset{T}{\operatorname{argmax}} P(T \mid W) & =\underset{T}{\operatorname{argmax}} \frac{P(T) \times P(W \mid T)}{P(W)}(\mathbf{1}) \\
& =\underset{T}{\operatorname{argmax}} P(T) \times P(W \mid T)(2)
\end{aligned}
$$

The term $P(W)$ is a constiant for all choices of $T$, and can thus be ignored when choosing the most probable $T$. We can further simplify the problem using tho assumption that words are independent of cach other given their tags. In Turkish we can use the adclitional simplification that $P^{\prime}\left(w_{i} \mid t_{i}\right)=1$ since $t_{i}$ includes the root form and all morphosyntactic features to uniquely detormine the word form. ${ }^{2}$ Since

\footnotetext{
2'That is, we assume that there is no morphological generation ambiguity. This is almost always true. 'T'here are a few word forms like gelirkene and nerde, which have the
}

in our case $I^{\prime}\left(w_{i} \mid t_{1}^{n}\right)=P\left(w_{i} \mid t_{i}\right)=1$, we can wite:

$$
P(W \mid T)=\prod_{i=1}^{n} P\left(w_{i} \mid t_{1}^{n}\right)=1
$$

and

$$
\underset{T}{\operatorname{argmax}} P\left(T^{\prime} \mid W\right)=\underset{T^{\prime}}{\operatorname{argmax}} P\left(T^{\prime}\right)
$$

Now,

$$
\begin{aligned}
P(T)= & P\left(t_{n} \mid t_{1}^{n-1}\right) \times P\left(t_{n-1} \mid t_{1}^{n-2}\right) \times \ldots \\
& \times P^{2}\left(t_{2} \mid t_{1}\right) \times P\left(t_{1}\right)
\end{aligned}
$$

Sinuplifying further with the trigram tagr model, we get:

$$
\begin{aligned}
P(T)= & P\left(t_{n} \mid t_{n-2}, t_{n-1}\right) \times \\
& I^{\prime}\left(t_{n-1} \mid t_{n-3}, t_{n-2}\right) \times \ldots \\
& I^{\prime}\left(t_{3} \mid t_{1}, t_{2}\right) \times P\left(t_{2} \mid t_{1}\right) \times P\left(t_{1}\right) \\
= & \prod_{i=1}^{n} P\left(t_{i} \mid t_{i-2}, t_{i-1}\right)
\end{aligned}
$$

where we define $P\left(t_{1} \mid t_{-1}, t_{0}\right)=P\left(t_{1}\right), P\left(t_{2} \mid t_{0}, t_{1}\right)=$ $P\left(t_{2} \mid t_{1}\right)$ to simplify the notation.

If wo consider morphological analyses as a sequence of root and IGs, each parse $t_{i}$ can be represented as $\left(r_{i}, I G_{i, 1}, \ldots, I G_{i, n_{i}}\right)$, where $n_{i}$ is the number of IGs in the $i^{\text {th }}$ word. ${ }^{3}$ This representation changes the problem as shown in Figure 1 where the chain rule has been used to factor ont the individual compononts.

'This formulation still suffers fiom the datia sparseness problem. 'To alleviate this, we make the following simplifying assumptions:

1. A root word depends only on the roots of the previous words, and is independent of the inflectional and derivational productions on them:

$$
\begin{array}{r}
P\left(r_{i} \mid\left(r_{i-2}, I G_{i-2,1}, \ldots, I G_{i-2, n_{i-2}}\right),\right. \\
\left.\left(r_{i-1}, I G_{i-1,1}, \ldots, I G_{i-1, n_{i-1}}\right)\right) \\
P\left(r_{i} \mid r_{i-2}, r_{i-1}\right)
\end{array}
$$

The intention here is that this will be useful in the disambiguation of the root word when a given form has morphological parses with different root words. So, for instance, for disambiguating the surface form adam with the following two parses:

same morphological parses with the word forms gelirken and nerede, respectively but are pronounced (and writen) slightly diflerently. These are rarely seon in written texts, and can thus be ignored.

${ }^{3}$ In our training and test data, the number of IGs in a word form is on the average 1.6, therefore, $n_{i}$ is usually $1 \mathrm{or} 2$. We have secen, occasionally, word forms with 5 or 6 inflectional groups. 


$$
\begin{aligned}
P\left(t_{i} \mid t_{1}^{i-1}\right)= & P\left(t_{i} \mid t_{i-2}, t_{i-1}\right) \\
= & P\left(\left(r_{i}, I G_{i, 1} \ldots I G_{i, n_{i}}\right) \mid\left(r_{i-2}, I G_{i-2,1} \ldots I G_{i-2, n_{i-2}}\right),\left(r_{i-1}, I G_{i-1,1} \ldots I G_{i-1, n_{i-1}}\right)\right) \\
= & P\left(r_{i} \mid\left(r_{i-2}, I G_{i-2,1} \ldots I G_{i-2, n_{i-2}}\right),\left(r_{i-1}, I G_{i-1,1} \ldots I G_{i-1, n_{i-1}}\right)\right) \times \\
& P\left(I G_{i, 1} \mid\left(r_{i-2}, I G_{i-2,1} \ldots I G_{i-2, n_{i-2}}\right),\left(r_{i-1}, I G_{i-1,1} \ldots I G_{i-1, n_{i-1}}\right), r_{i}\right) \times \\
& \ldots \times \\
& P\left(I G_{i, n_{i}} \mid\left(r_{i-2}, I G_{i-2,1} \ldots I G_{i-2, n_{i-2}}\right),\left(r_{i-1}, I G_{i-1,1} \ldots I G_{i-1, n_{i-1}}\right), r_{i}, I G_{i, 1}, \ldots, I G_{i, n_{i-1}}\right)
\end{aligned}
$$

Figure 1: Equation for morphological disambiguation when tags are decomposed into inflectional groups.

\section{(a) adam+Noun+A3sg+Pnon+Nom ( an ) \\ (b) ada+Noun+A3sg+P1sg+Nom (my island)}

in the noun phrase kirmezt kazakl adam (the man with a red sweater), only the roots (along with the part-of-specch of the root) of the previous words will be used to select the right root. Note that the selection of the root has some impact on what the next IG in the word is, but wo assume that IGs are determined by the syntactic context and not by the root.

2. An interesting observation that we can make about Turkish is that when a word is considcred as a sequence of IGs, syntactic relations are between the last IG of a (dependent) word and with some (including the last) IG of the (head) word on the right (with minor exceptions) (Oflazer, 1999).

Based on these assumptions and the equation in Figure 1, we define three models, all of which are based on word level trigrams:

1. Model 1: The presence of IGs in a word only depends on the final IGs of the previous words. This model ignores any morphotactical rolation between an IG and any previous IG in the same word.

2. Model 2: The presence of IGs in a word only depends on the final IGs of the previous words and the previous IG in the same word. In this model, we consider morphotactical relations and assume that an IG (cxcept the first one) in a word form has some dependency on the previous IG. Given that on the average a word has about 1.6 IGs, IG bigrams should be sufficient.

3. Model 3: This is the same as Model 2, except that the dependence with the previous IG in a word is assumed to be independent of the dependence on the final IGs of the previous words. This allows the formulation to separate the contributions of the morphotactics and syntax.

The equations for these models are shown in Figure 2. We also have built a bascline model based on the standard dofinition of the tagging problem in Equation 2. For the baseline, we have assumed that the part of the morphological analysis after the root word is the tag in the conventional sense (and the assumption that $P\left(w_{i} \mid t_{i}\right)=1$ no longer holds $)$.

\section{$5 \quad$ Experiments and Results}

To cvaluate our models, wo first trained our models and then tried to morphologically disambiguatc our test data. For statistical modeling we used SRILM - the SRI language modeling toolkit (Stolcke, 1999).

Both the test data and training data were collected from the wob resources of a Turkish daily newspaper. The tokens were analyzed using the morphological analyzer, developed by Oflazer (1994). The ambiguity of the training data was then reduced from 1.75 to 1.55 using a preprocessor, that disambiguates lexicalized and non-lexicalized collocations and romoves cortain obviously impossible parses, and tries to analyze unknown words with an unknown word processor. The training data consists of the unambiguous sequences (US) consisting of about $650 \mathrm{~K}$ tokens in a corpus of 1 million tokens, and two sets of manually disambiguated corpora of 12,000 and 20,000 tokens. The idea of using unambiguous sequences is similar to Brill's work on unsupervised learning of disambiguation rules for POS tagging (1995b).

The test data consists of 2763 tokens, 935 ( $\approx 34 \%$ ) of which have more than one morphological analysis after preprocessing. The ambiguity of the test data was reduced from 1.74 to 1.53 after preprocessing.

As our evaluation metric, we used accuracy defincd as follows:

$$
\text { accuracy }=\frac{\# \text { of correct parses }}{\# \text { of tokens }} \times 100
$$

The accuracy results are given in Table 4. For all cases, our models performed better than bascline tag model. As expected, the tag model suffered considerably from data sparseness. Using all of our training data, we achieved an accuracy of $93.95 \%$, which is $2.57 \%$ points better than the tag model trained using the same amount of data. Models 2 and 3 gave 
In all three models we assune that roots and IGs are independent.

Model 1: This model assumes that an IG in a word depends on the last IGs of the two previous words.

$$
\begin{array}{r}
P\left(I G_{i, k} \mid\left(r_{i-2}, I G_{i-2,1} \ldots I G_{i-2, n_{i-2}}\right),\left(r_{i-1}, I G_{i-1,1}, \ldots, I G_{i-1, n_{i-1}}\right), r_{i}, I G_{i, 1}, \ldots, I G_{i, k-1}\right) \\
P\left(I G_{i, k} \mid I G_{i-2, n_{i-2}}, I G_{i-1, n_{i-1}}\right)
\end{array}
$$

Therefore,

$$
P\left(t_{i} \mid t_{i-2}, t_{i-1}\right)=P\left(r_{i} \mid r_{i-2}, r_{i-1}\right) \times \prod_{k=1}^{n_{i}} P\left(I G_{i, k} \mid I G_{i-2, n_{i-2}}, I G_{i-1, n_{i-1}}\right)
$$

Model 2: The model assumes that in addition to the dependencies in Model 1, an IG also depends on the previous IG in the same word.

$$
\begin{array}{r}
P\left(I G_{i, k} \mid\left(r_{i-2}, I G_{i-2,1} \ldots I G_{i-2, n_{i-2}}\right),\left(r_{i-1}, I G_{i-1,1}, \ldots, I G_{i-1, n_{i-1}}\right), r_{i}, I G_{i, 1}, \ldots, I G_{i, k-1}\right) \\
I\left(I G_{i, k} \mid I G_{i-2, n_{i-2}:} I G_{i-1, n_{i-1}}, I_{\left.\mathbf{G}_{\mathbf{i}, \mathrm{k}-1}\right)}\right)
\end{array}
$$

Therefore,

$$
P\left(t_{i} \mid t_{i-2}, t_{i-1}\right)=P\left(r_{i} \mid r_{i-2,2}, r_{i-1}\right) \times \prod_{k=1}^{n_{i}} P\left(I G_{i, k} \mid I G_{i-2, n_{i-2}}, I G_{i-1, n_{i-1}}, \mathbf{I G}_{\mathrm{i}, \mathbf{k}-1}\right)
$$

Model 3: This is same as Model 2, except the morphotactic and syntactic dependencies are considered to be independent.

$$
\begin{aligned}
& P\left(I G_{i, k} \mid\left(r_{i-2}, I G_{i-2,1} \ldots I G_{i-2, n_{i-2}}\right),\left(r_{i-1}, I G_{i-1,1}, \ldots, I G_{i-1, n_{i-1}}\right), r_{i}, I G_{i, 1}, \ldots, I G_{i, k-1}\right)= \\
& I\left(I G_{i, k} \mid I G_{i-2, n_{i-2}}, I G_{i-1, n_{i-1}}, I G_{i, k-1}\right) \\
& P\left(I G_{i, k} \mid I G_{i \cdot 2, n_{i-2}}, I G_{i-1, n_{i-1}}, I G_{i, k-1}\right)=I^{\prime}\left(I G_{i, k} \mid I G_{i \cdots 2, n_{i-2}}, I G_{i-1, n_{i-1}}\right) \times \frac{P\left(I G_{i, k} \mid I G_{i, k-1}\right)}{P\left(I G_{i, k}\right)}
\end{aligned}
$$

Therefore,

$$
P\left(t_{i} \mid t_{i-2}, t_{i-1}\right)=P\left(r_{i} \mid r_{i-2}, r_{i-1}\right) \times \prod_{k=1}^{n_{i}}\left(P\left(I G_{i, k} \mid I G_{i-2, n_{i-2}}, I G_{i-1, n_{i-1}}\right) \times \frac{P\left(I G_{i, k} \mid I G_{i, k-1}\right)}{I^{\prime}\left(I G_{i, k}\right)}\right)
$$

In order to simplify the notation, we have defined the following:

$$
\begin{aligned}
& P\left(r_{1} \mid r_{-1}, r_{0}\right)=P\left(r_{1}\right) \quad P\left(I G_{1, k} \mid I G_{-1, n_{-1}}, I G_{0, n_{0}}\right)=P\left(I G_{1, k}\right) \\
& P\left(r_{2} \mid r_{0}, r_{1}\right)=P\left(r_{2} \mid r_{1}\right) \quad P\left(I G_{2, l} \mid I G^{\prime}\left(r_{1, n_{0}, I}, I G_{1, n_{1}}\right)=P^{\prime}\left(I G_{2, l} \mid I G_{1, n_{1}}\right)\right. \\
& P\left(I G_{i, 1} \mid I G_{i-2, n_{i-2}}, I G_{i-1, n_{i-1}}, I G_{i, 0}\right)=P\left(I G_{i, 1} \mid I G_{i-2, n_{i-2}}, I G_{i-1, n_{i-1}}\right) \\
& P\left(I G_{1, k} \mid I G_{-1, n_{-1}}, I G_{0, n_{0}}, I G_{1, k-1}\right)=P\left(I G_{1, k} \mid I G_{1, k-1}\right) \\
& P\left(I G_{2, l} \mid I G_{0, n_{0}}, I G_{1, n_{1}}, I G_{2, l-1}\right)=I\left(I G_{2, l} \mid I G_{1, n_{1}}, I G_{2, l-1}\right) \\
& P\left(I G_{2,1} \mid I G_{1, n_{1}}, I G_{2,0}\right)=P\left(I G_{2,1} \mid I G_{1, n_{1}}\right) \\
& P\left(I G_{i, 1} \mid I G_{i, 0}\right) \quad=P\left(I G_{i, 1}\right)
\end{aligned}
$$

for $k=1,2, \ldots, n_{4}, l=1,2, \ldots, n_{2}$, and $i=1,2, \ldots, n$.

Figure 2: Equations for Models 1,2 , and 3. 


\begin{tabular}{|l|c|c|c|c|c|}
\hline Training Data & $\begin{array}{c}\text { Tag Model } \\
\text { (Baseline) }\end{array}$ & Model 1 & $\begin{array}{c}\text { Model 1 } \\
\text { (Bigram) }\end{array}$ & Model 2 & Model 3 \\
\hline Unambiguous sequences (US) & $86.75 \%$ & $88.21 \%$ & $89.06 \%$ & $87.01 \%$ & $87.19 \%$ \\
US + 12,000 words & $91.34 \%$ & $93.52 \%$ & $93.34 \%$ & $92.43 \%$ & $92.72 \%$ \\
US + 32,000 words & $91.34 \%$ & $93.95 \%$ & $93.56 \%$ & $92.87 \%$ & $92.94 \%$ \\
\hline
\end{tabular}

Table 4: Accuracy results for different models.

similar results, Model 2 suffered from data sparseness slightly more than Model 3 , as expected.

Surprisingly, the bigram version of Model 1 (i.c., Equation (7), but with bigrams in root and IG models), also performs quite well. If we consider just the syntactically relevant morphological features and ignore any semantic features that we mark in morphology, the accuracy increases a bit further. These stem from two properties of Turkish: Most Turkish root words also have a proper noun reading, when written with the first letter capitalized. ${ }^{4}$ Wo count it as an error if the tagger does not get the correct proper noun marking, for a proper noun. But this is usually impossible especially at the beginning of sentences where the tagger can not exploit capitalization and has to back-off to a lower-order model. In almost all of such cases, all syntactically rolevant morphosyntactic features except the proper noun marking are actually correct. Another important case is the pronom $o$, which has both personal pronoun (s/he) and domonstrative pronoun roadings (it) (in addition to a syntactically distinct determiner reading (that)). Resolution of this is always by semantic considerations. When we count as correct any crrors involving such semantic marker cases, wo get an accuracy of $95.07 \%$ with the best case (cf. $93.91 \%$ of the Model 1). This is slightly better than the precision figures that is reported carlicr on morphological disambiguation of Turkish nsing constraintbased techniques (Oflazer and Tür, 1997). Our results are slightly better than the results on Crech of Hajič and Hladká (1998). Megyesi (1999) reports a $95.53 \%$ accuracy on Hungarian (a language whose features relevant to this task are very close to those of Turkish), with just the POS tags being correct. In our model this corresponds to the root and the POS tag of the last IG bcing correct and the accuracy of our best model with this assumption is $96.07 \%$. When POS tags and subtags are considered, the reported accuracy for Hungarian is $91.94 \%$ while the corresponding accuracy in our case is $95.07 \%$. We can also note that the results presented by Ezciza et al. (1998) for Basque are better than ours. The main reason for this is that they employ a much more sophisticated (compared to our preprocessor)

\footnotetext{
${ }^{4}$ In fact, any word form is a potential first name or a last
}

constraint-grammar based system which improves precision without reducing recall. Statistical techniques applicel after this disambiguation yield a better accuracy compared to starting from a more ambiguous initial state.

Since our models assumed that wo have independent models for disambiguating the root words, and the IGs, we ran experiments to see the contribution of the individual models. Table 5 summarizes the accuracy results of the individual models for the best casc (Modol 1 in Table 4.)

\begin{tabular}{|l|r|}
\hline Model & Accuracy \\
\hline IG Model & $92.08 \%$ \\
Root Model & $80.36 \%$ \\
Combined Model & $93.95 \%$ \\
\hline
\end{tabular}

Table 5: 'The contribution of the individual models for the best case.

There are quite a number of classes of words which are always ambiguous and the preprocessing that we have cmployed in creating the unambiguous sequences can never resolve these cases. Thus statistical models trained using only the unambiguous sequences as the training data do not handle these ambiguous cases at all. This is why the accuracy results with only unambiguous sequences are significantly lower (row 1 in Table 4). The manually disambiguated training sets have such ambiguitics resolved, so those models perform much better.

An analysis of the errors indicates the following: In $15 \%$ of the errors, the last IG of the word is incorrect but the root and the rest of the IGs, if any, are correct. In $3 \%$ of the errors, the last IG of the word is correct but the either the root or some of the previous IGs are incorrect. In $82 \%$ of the crrors, neither the last IG nor any of the previous IGs are correct. Along a different dimension, in about $51 \%$ of the crrors, the root and its part-of-specel are not determined correctly, while in $84 \%$ of the errors, the root and the first IG combination is not correctly determined. 


\section{Conclusions}

Wo have presented an approach to statistical modleling for agghutinative languages, especially those having productive derivational phenomenal. (Our approach essentially involves breaking up the full norphological analysis across derivational boundaries and treating the components as subtags, and then detemining the correct sequence of tags via statistical tochniques. This, to om knowleclge, is the first detailed attempt in statistical modoling of agglntinative langudges and can certanly bo applied to other such langllages like Hungarian and Fimish with productive derivational morphology.

\section{Acknowledgments}

Wo thank Audreas Stolcke of SIR] S'IAR I a bor provicling us with the langnage modeling toolkit and for very helpful discussions on this work. I.iz Shuiberg of SRI SIAR, Labs, and Bilge Say of Middle Itast Iechnical University Informatics lnstitute, provided helpful insights and comnnonts.

\section{References}

Fric: 13rill. 1995a. Transformation-based crordriven loanning and natural language procossing: A case study in part-of-spech tagging. Computational Linguistics, 21(4):543-566, Docember.

Eric: Brill. 1995b. Unsupervised learning of disambiguation rules for part of speech talgging. In Procecdings of the Third Workshop on Very Iarge Corpora, Cambridge, MA, June.

Kenucth W. Church. 1988. A stochastic parts program and a nom phraso parser for monestricted text. In Procedings of the Second Conference on Applied Natural Language Processing, Austin, Texas.

Doug Cutting, Julian Kupiec, Jan Pedersen, and Penclope Sibun. 1992. A practical part-of-speech tagger. In Procedings of the Third Conference on Aprlied Natural Language Processing, Tronto, Italy.

N. Ezeiza, I. Alogria, J. M. Arriola, R. Urizar, and 1. Aduri\% 1998. Combining stochastic and rulebased methods for disambiguation in agghtutinative languages. In Procedings of the $36^{t h}$ Annual Mecting of the Association for Computational Linguistics and $17^{\text {th }}$ International Conference on Computational Linguistics, pages 379 384, Montreal, Quchec, Canada, August.

Jan Hajič and Barbora Hladkí. 1998. Tagging inflective languages: Prediction of morphological categories for a rich, structured tagset. In Procedings of COLING/ACL'98, pages 483-490, Montrcal, Canada, August.

Jorge Hankamer, 1989. Lexical Representation and Process, chapter Morphological Parsing and the Lexicon. The MIT Press. lred Karlsson, Atro Voutilainen, Juha Heikkilä, and Arto Anttila. 1995. Constraint Grommar-A Language-Independent System for Parsing Unrestricted Text. Mouton do Gruyter.

Moshe Levinger, Uzzi Ornan, and Alon Itai. 1995. learning mospho-lexical probabilities from an untagged corpus with an application to Hebrew. Computational Linguistics, 21(3):383 404, September.

Bcáta Megyesi. 1.999. Inproving Brill's POS tagger for an agglutinative language. In Pascale rung and Joe Zhou, editors, Proceedings of the Joint SIGDAT Conference on Enpirical Methods in Natural Language Processing and Very Large Corpora, pages 275-284, College Park, Marylanc, USA, Junc.

Konal Oflazer and İker Kuruöz. 1994. Tagging and morphological disambiguation of Thurkish toxt. In Procedings of the $4^{\text {th }}$ Applied Natural Language Processing Conference, pages 144 -149. ACL, OCtolorer.

Kemal Oflazer and Gökhan Tlür. 1996. Combining hand-coafted rules and unsupervised learning in constratint,-based morphological disambiguattion. In Frice 13rill and Konneth Chnuch, editors, Procedings of the ACI,STGI)AT' Conference on Empirical Mothods in Natural Language Processing.

Kemal Oflazer and Gökhan Tür. 1997. Morplological disambiguation by voting constraints. In Procecdings of the $35^{\text {th }}$ Annual Meeting of the Associntion for Computational Linquistics (ACI'?7/WACL'97), Madrid, Spain, July.

Kemal oflaver, Dilek 7. Hakkani-Tiü, and Gökhan 'T'ür. 1999. I) esign for a Turkish trecobank. In P'roceedings of Workshop on Linguistically Interpreted Corpora, at EACJ'99, Bergen, Norway, June.

Kemal Oflazer. 1994. Two-level description of 'Turkish morphology. Literary and linguistic: Computing, $9(2): 137 \cdots 148$.

Kemal Oflazer. 1999. Dependency parsing with an extended finite state approach. In Proceedings of the 37th Annual Meeting of the Association for Computational Linguisties, College P'ark, Maryland, June.

Andreas Stolcke. 1999. SRILM-tho SRI language modoling toolkit. http://www.speech.sri.com/ projects/srilm/. 